\title{
Stochastic Predator-Prey System Subject to Lévy Jumps
}

\author{
Xinzhu Meng ${ }^{1,2}$ and Xiaohong Wang ${ }^{1,2}$ \\ ${ }^{1}$ State Key Laboratory of Mining Disaster Prevention and Control Cofounded by Shandong Province and the Ministry of Science and \\ Technology, Shandong University of Science and Technology, Qingdao 266590, China \\ ${ }^{2}$ College of Mathematics and Systems Science, Shandong University of Science and Technology, Qingdao 266590, China
}

Correspondence should be addressed to Xinzhu Meng; mxz721106@sdust.edu.cn

Received 6 January 2016; Accepted 13 March 2016

Academic Editor: Elvan Akın

Copyright (C) 2016 X. Meng and X. Wang. This is an open access article distributed under the Creative Commons Attribution License, which permits unrestricted use, distribution, and reproduction in any medium, provided the original work is properly cited.

\begin{abstract}
This paper investigates a new nonautonomous impulsive stochastic predator-prey system with the omnivorous predator. First, we show that the system has a unique global positive solution for any given initial positive value. Second, the extinction of the system under some appropriate conditions is explored. In addition, we obtain the sufficient conditions for almost sure permanence in mean and stochastic permanence of the system by using the theory of impulsive stochastic differential equations. Finally, we discuss the biological implications of the main results and show that the large noise can make the system go extinct. Simulations are also carried out to illustrate our theoretical analysis conclusions.
\end{abstract}

\section{Introduction}

Omnivory is considered as a common ecological phenomenon in the natural world. Omnivorous predator feeds on both animal prey and plant, so the intrinsic growth rate for predator should be positive. For example, the giant panda is omnivorous animal, since it can eat both meat and plant such as bamboo. With the development of the economy, pollution is becoming more and more serious. Thus pollution models have widely attracted the focus of the people [15]. A deterministic predator-prey system with omnivorous predator in an impulsive polluted environment takes the following form:

$$
\begin{aligned}
& d x(t) \\
& \quad=x(t)\left(r_{1}(t)-d_{1} c_{0}(t)-a_{1}(t) x(t)-\beta(t) y(t)\right) d t, \\
& d y(t) \\
& \quad=y(t)\left(r_{2}(t)-d_{2} c_{0}(t)-a_{2}(t) y(t)+\beta(t) x(t)\right) d t, \\
& \dot{c}_{0}(t)=k c_{e}(t)-g c_{0}(t)-m c_{0}(t), \\
& \dot{c}_{e}(t)=-h c_{e}(t),
\end{aligned}
$$

$$
\begin{aligned}
& \Delta x\left(\tau_{k}\right)=\Delta y\left(\tau_{k}\right)=\Delta c_{0}\left(\tau_{k}\right)=0, \\
& \begin{array}{ll}
\Delta c_{e}\left(\tau_{k}\right)=u, \\
\\
\tau_{k}=k \tau, k=0,1, \ldots,
\end{array}
\end{aligned}
$$

where $\Delta x\left(\tau_{k}\right)=x\left(\tau_{k}^{+}\right)-x\left(\tau_{k}\right), \Delta y\left(\tau_{k}\right)=y\left(\tau_{k}^{+}\right)-y\left(\tau_{k}\right)$, $\Delta c_{0}\left(\tau_{k}\right)=c_{0}\left(\tau_{k}^{+}\right)-c_{0}\left(\tau_{k}\right), c_{e}\left(\tau_{k}\right)=c_{e}\left(\tau_{k}^{+}\right)-c_{e}\left(\tau_{k}\right), \delta_{i}(i=$ $1,2), k, g, m, h$, and $u$ are positive constants, $x(t)$ and $y(t)$ denote prey and omnivorous predator densities, and $c_{0}(t)$ and $c_{e}(t)$ denote the concentrations of the toxicant in the organism and in the environment, respectively. $r_{i}(t), a_{i}(t)(i=1,2)$, and $\beta(t)$ are all positive bounded continuous functions on $R_{+}:=[0,+\infty), r_{1}(t)$ and $r_{2}(t)$ represent the prey intrinsic growth rate and the predator intrinsic growth rate, respectively, $a_{i}(t)(i=1,2)$ are the density-dependent coefficients of the prey and the predator, $\beta(t)$ is the capturing rate of the predator, $d_{1}$ and $d_{2}$ are damage rates of the prey and predator by the toxicant, respectively, $k$ represents environmental toxicant uptake rate per unit mass organism, $g$ and $m$ are organismal net ingestion and depuration rates of toxicant, respectively, $h$ denotes the loss rate of toxicant from the environment itself by volatilization, and $u$ is the amount of pulsed input concentration of the toxicant at each $\tau$. 
System (1) is a deterministic model, where all parameters in the model are deterministic. However, there are some limitations in mathematical model from a biological viewpoint. Therefore, it is significant to study the effects of noises on population systems [6-9]. There are many kinds of environmental noises. First, we assume that the toxicant uptake rates are disturbed by white noise. If we still let $d_{i} c_{0}(t)(i=1,2)$ represent the toxicant uptake rates, then $d_{1} c_{0}(t)$ and $d_{2} c_{0}(t)$ can be replaced by

$$
\begin{aligned}
& d_{1} c_{0}(t) \longrightarrow d_{1} c_{0}(t)+\sigma_{1}(t) \dot{B}_{1}(t), \\
& d_{2} c_{0}(t) \longrightarrow d_{2} c_{0}(t)+\sigma_{2}(t) \dot{B}_{2}(t),
\end{aligned}
$$

where $\dot{B}_{i}(t)(i=1,2)$ are white noises and $\sigma_{i}(t)(i=1,2)$ are the intensities of the white noises, which are bounded continuous functions on $[0,+\infty)$. Then we obtain the following stochastic system with impulsive toxicant input in a polluted environment:

$$
\begin{aligned}
& d x(t) \\
& =x(t)\left(r_{1}(t)-d_{1} c_{0}(t)-a_{1}(t) x(t)-\beta(t) y(t)\right) d t \\
& -\sigma_{1}(t) x(t) d B_{1}(t), \\
& d y(t) \\
& =y(t)\left(r_{2}(t)-d_{2} c_{0}(t)-a_{2}(t) y(t)+\beta(t) x(t)\right) d t \\
& -\sigma_{2}(t) y(t) d B_{2}(t) \text {, } \\
& \dot{c}_{0}(t)=k c_{e}(t)-g c_{0}(t)-m c_{0}(t), \\
& \dot{c}_{e}(t)=-h c_{e}(t) \text {, } \\
& t \neq \tau_{k} \\
& \Delta x\left(\tau_{k}\right)=\Delta y\left(\tau_{k}\right)=\Delta c_{0}\left(\tau_{k}\right)=0, \\
& \Delta c_{e}\left(\tau_{k}\right)=u,
\end{aligned}
$$

where $B_{i}(t)$ are mutually independent standard Brownian motions defined on a complete probability space $\left(\Omega, \mathscr{F},\{\mathscr{F}\}_{t \geq 0}, \mathscr{P}\right)$.

On the other hand, populations may be affected by sudden environmental fluctuations, such as severe weather, earthquakes, floods, and epidemics. Brownian motion cannot describe these phenomena better, so it is very important to introduce Lévy noise into the population system [10]. There are many researches about autonomous stochastic predatorprey system with Lévy jumps [11, 12].

Inspired by these, we focus on nonautonomous impulsive stochastic predator-prey system with white noises and Lévy jumps

$$
\begin{aligned}
d x( & \\
= & x(t)\left(r_{1}(t)-d_{1} c_{0}(t)-a_{1}(t) x(t)-\beta(t) y(t)\right) d t \\
& -\sigma_{1}(t) x(t) d B_{1}(t) \\
& +\int_{\mathbb{Y}} x\left(t^{-}\right) \gamma_{1}(t, u) \widetilde{N}(d t, d u),
\end{aligned}
$$

$$
\begin{aligned}
& d y(t) \\
& =y(t)\left(r_{2}(t)-d_{2} c_{0}(t)-a_{2}(t) y(t)+\beta(t) x(t)\right) d t \\
& -\sigma_{2}(t) y(t) d B_{2}(t) \\
& +\int_{\mathbb{Y}} y\left(t^{-}\right) \gamma_{2}(t, u) \widetilde{N}(d t, d u), \\
& \dot{c}_{0}(t)=k c_{e}(t)-g c_{0}(t)-m c_{0}(t), \\
& \dot{c}_{e}(t)=-h c_{e}(t) \text {, } \\
& \Delta x\left(\tau_{k}\right)=\Delta y\left(\tau_{k}\right)=\Delta c_{0}\left(\tau_{k}\right)=0, \\
& \Delta c_{e}\left(\tau_{k}\right)=u, \\
& t \neq \tau_{k}, \\
& \tau_{k}=k \tau, k=0,1, \ldots,
\end{aligned}
$$

where $x\left(t^{-}\right)$and $y\left(t^{-}\right)$represent the left limit of $x(t)$ and $y(t)$, respectively, $N(d t, d u)$ is a Poisson counting measure with characteristic measure $v$ on a measurable bounded subset $\mathbb{Y}$ of $(0, \infty)$ with $\nu(\mathbb{Y})<\infty$, and $B_{i}(i=1,2)$ are independent of $N$. The Poisson counting measure is represented by $\widetilde{N}(d t, d u):=N(d t, d u)-v(d u) d t ; \gamma_{i}>$ $-1, \gamma_{i}: \mathbb{R}_{+} \times \mathbb{Y} \rightarrow \mathbb{R}(i=1,2)$ are continuous functions on $[0,+\infty)$, which are assumed to be periodic with period $\tau>0$. Other parameters are defined as in system (1).

The paper is arranged as follows. In Section 2, we prove that system (4) has a global positive solution. Section 3 shows the main result; in Section 3.1 we prove the extinction of system (4). We also examine almost sure permanence in mean and the stochastic permanence of the system in Sections 3.2 and 3.3. Finally we present some simulations and conclusions to close the paper in Section 4.

\section{Notations and Global Positive Solution}

For the purpose of convenience, we introduce some notions and some lemmas which will be used for our main results. We throughout this paper assume that $x(t), y(t)$, and $c_{0}(t)$ are continuous at $t=k \tau$, and $c_{e}(t)$ is left continuous at $t=k \tau$ and $c_{e}\left(k \tau^{+}\right)=\lim _{t \rightarrow k \tau^{+}} c_{e}(t)$ and let $\left(\Omega, \mathscr{F},\{\mathscr{F}\}_{t \geq 0}, \mathscr{P}\right)$ be a complete probability space with a filtration $\left\{\mathscr{F}_{t}\right\}_{t \geq 0}$ satisfying the usual conditions (i.e., it is increasing and right continuous while $\mathscr{F}_{0}$ contains all $\mathscr{P}$-null sets). Further assume that $B(t)$ is a scalar Brownian motion defined on the complete probability space $\Omega$.

We denote $\mathbb{R}_{+}^{2}$ as the positive cone in $\mathbb{R}^{2}$; that is, $\mathbb{R}_{+}^{2}=$ $\left\{z=\left(z_{1}, z_{2}\right) \in \mathbb{R}^{2} \mid z_{i}>0, i=1,2\right\}$. If $f(t)$ is a bounded continuous function on $[0,+\infty)$, we define

$$
\begin{aligned}
\langle f(t)\rangle & =\frac{1}{t} \int_{0}^{t} f(t) d t, \\
f^{M} & =\max _{t \in[0,+\infty)} f(t),
\end{aligned}
$$




$$
\begin{aligned}
& f^{L}=\min _{t \in[0,+\infty)} f(t), \\
& f^{*}=\limsup _{t \rightarrow+\infty} f(t), \\
& f_{*}=\liminf _{t \rightarrow+\infty} f(t) .
\end{aligned}
$$

Now we give some basic properties of the following subsystem of systems (1) and (4):

$$
\begin{aligned}
& d c_{0}(t)=\left(k c_{e}(t)-g c_{0}(t)-m c_{0}(t)\right) d t \\
& d c_{e}(t)=-h c_{e}(t) d t
\end{aligned}
$$$$
t \neq k \tau, k \in Z^{+}
$$

$$
\begin{aligned}
& \Delta c_{0}(t)=0, \\
& \Delta c_{e}(t)=u,
\end{aligned}
$$$$
t=k \tau, k \in Z^{+}
$$

Lemma 1 (see [3]). System (6) has a unique positive $\tau$-periodic solution $\left(c_{0}^{*}(t), c_{e}^{*}(t)\right)^{T}$ which is globally asymptotically stable. Moreover, $c_{0}(t)>c_{0}^{*}(t), c_{e}(t)>c_{e}^{*}(t)$ for all $t \geq 0$ if $c_{0}(0)>$ $c_{0}^{*}(0), c_{e}(0)>c_{e}^{*}(0)$, where

$$
\begin{aligned}
c_{0}^{*}(t)= & c_{0}^{*}(0) e^{-(g+m)(t-n \tau)} \\
& +\frac{k u\left(e^{-(g+m)(t-n \tau)}-e^{-h(t-n \tau)}\right)}{(h-g-m)\left(1-e^{-h \tau}\right)}, \\
c_{e}^{*}(t)= & \frac{u e^{-h(t-n \tau)}}{1-e^{-h \tau}}, \\
c_{0}^{*}(0)= & \frac{k u\left(e^{-(g+m) \tau}-e^{-h \tau}\right)}{(h-g-m)\left(1-e^{-(g+m) \tau}\right)\left(1-e^{-h \tau}\right)}, \\
c_{e}^{*}(0)= & \frac{u}{1-e^{-h \tau}},
\end{aligned}
$$

for $t \in(k \tau,(k+1) \tau]$ and $k \in Z^{+}$.

Lemma 2. For any positive solution $\left(c_{0}(t), c_{e}(t)\right)$ of system (1) or (4) with initial value $\left(c_{0}(0), c_{e}\left(0^{+}\right)\right) \in \mathbb{R}_{+}^{2}$, one has

$$
\lim _{t \rightarrow+\infty}\left\langle c_{0}(t)\right\rangle=\frac{k u}{h(g+m) \tau} \triangleq \overline{c_{0}} .
$$

Proof. Through a simple calculation, we can get

$$
\int_{n \tau}^{(n+1) \tau} c_{0}^{*}(t) d t=\frac{k u}{h(g+m)} .
$$

Moreover, since $c_{0}^{*}(t)$ is a periodic function, we have

$$
\begin{aligned}
\lim _{t \rightarrow+\infty}\left\langle c_{0}(t)\right\rangle^{*} & \leq \lim _{n \rightarrow+\infty} \frac{1}{n \tau} \int_{0}^{(n+1) \tau} c_{0}^{*}(t) d t \\
& =\lim _{n \rightarrow+\infty} \frac{n+1}{n \tau} \int_{n \tau}^{(n+1) \tau} c_{0}^{*}(t) d t \\
& =\frac{k u}{h(g+m) \tau}, \\
\lim _{t \rightarrow+\infty}\left\langle c_{0}(t)\right\rangle_{*} & \geq \lim _{n \rightarrow+\infty} \frac{1}{(n+1) \tau} \int_{0}^{n \tau} c_{0}^{*}(t) d t \\
& =\lim _{n \rightarrow+\infty} \frac{n}{(n+1) \tau} \int_{(n-1) \tau}^{n \tau} c_{0}^{*}(t) d t \\
& =\frac{k u}{h(g+m) \tau} .
\end{aligned}
$$

Hence one can observe that

$$
\lim _{t \rightarrow+\infty}\left\langle c_{0}(t)\right\rangle=\frac{k u}{h(g+m) \tau} \triangleq \overline{c_{0}} .
$$

Then we show an assumption which will be used in the following proof.

Assumption 3. There exists a bounded continuous function $c_{i}(t)$ such that

$$
\int_{\mathbb{Y}}\left[\gamma_{i}-\ln \left(1+\gamma_{i}\right)\right] \nu(d u) \leq c_{i}(t), \quad(i=1,2) .
$$

Theorem 4. For any given initial value $(x(0), y(0)) \in \mathbb{R}_{+}^{2}$, there is a unique solution $X(t)=(x(t), y(t))$ of (4) and the solution will remain in $\mathbb{R}_{+}^{2}$ with probability 1 ; that is, $X(t) \in \mathbb{R}_{+}^{2}$ for all $t \geq 0$ almost surely.

Proof. The coefficient of (4) is locally Lipschitz continuous, and so, for any given initial value $(x(0), y(0)) \in \mathbb{R}_{+}^{2}$, there is a unique local solution $(x(t), y(t))$ for $t \in\left[0, \tau_{e}\right]$, where $\tau_{e}$ is the explosion time. To demonstrate that this solution is global, we need to show that $\tau_{e}=\infty$ a.s. Let $k_{0}>0$ be sufficiently large for $x_{0} \in\left[1 / k_{0}, k_{0}\right]$ and $y_{0} \in\left[1 / k_{0}, k_{0}\right]$. For each integer $k \geq k_{0}$, define the stopping time

$$
\begin{aligned}
& \tau_{k} \\
& =\inf \left\{t \in\left[0, \tau_{e}\right]: x(t) \bar{\epsilon}\left(\frac{1}{k}, k\right) \text { or } y(t) \bar{\epsilon}\left(\frac{1}{k}, k\right)\right\},
\end{aligned}
$$

where we set inf $\emptyset=\infty$ ( $\emptyset$ denotes the empty set). Obviously, $\tau_{k}$ is increasing as $k \rightarrow+\infty$. Set $\tau_{\infty}=\lim _{k \rightarrow+\infty} \tau_{k}$; thus $\tau_{\infty} \leq$ $\tau_{e}$ a.s., so we just need to demonstrate that $\tau_{\infty}=\infty$. If $\tau_{\infty}=$ $\infty$ is not true, then there exist two constants $T>0$ and $\varepsilon \in$ $(0,1)$ such that $P\left\{\tau_{\infty} \leq T\right\}>\varepsilon$. Thus there is an integer $k_{1} \geq$ $k_{0}$ such that $P\left\{\tau_{k} \leq T\right\} \geq \varepsilon$ for all $k \geq k_{1}$.

Define a function $V: \mathbb{R}_{+}^{2} \rightarrow \mathbb{R}_{+}$as follows:

$$
V(x, y)=x-1-\ln x+y-1-\ln y .
$$


Let $T>0$ be arbitrary. Applying Itô’s formula and Assumption 3 leads to

$$
\begin{aligned}
& d V(x, y)=L V d t-(x-1) \sigma_{1}(t) d B_{1}(t)-(y-1) \\
& \quad \cdot \sigma_{2}(t) d B_{2}(t) \\
& \quad+\int_{\mathbb{V}}\left[x\left(t^{-}\right) \gamma_{1}(t, u)-\ln \left(1+\gamma_{1}(t, u)\right)\right] \widetilde{N}(d t, d u) \\
& \quad+\int_{\mathbb{V}}\left[y\left(t^{-}\right) \gamma_{2}(t, u)-\ln \left(1+\gamma_{2}(t, u)\right)\right] \widetilde{N}(d t, d u),
\end{aligned}
$$

where

$$
\begin{array}{r}
L V=(x-1)\left(r_{1}(t)-d_{1} c_{0}(t)-a_{1}(t) x-\beta(t) y\right) \\
+\frac{1}{2} \sigma_{1}^{2}(t)+(y-1)\left(r_{2}(t)-d_{2} c_{0}(t)-a_{1}(t) y\right.
\end{array}
$$

$$
\begin{aligned}
& +\beta(t) x)+\frac{1}{2} \sigma_{2}^{2}(t) \\
& +\int_{\mathbb{Y}}\left[\gamma_{1}(t, u)-\ln \left(1+\gamma_{1}(t, u)\right)\right] v(d u) \\
& +\int_{\mathbb{V}}\left[\gamma_{2}(t, u)-\ln \left(1+\gamma_{2}(t, u)\right)\right] v(d u) \leq-a_{1}(t) \\
& \cdot x^{2}+\left(a_{1}(t)+r_{1}(t)-d_{1} c_{0}(t)-\beta(t)\right) x-r_{1}(t) \\
& +d_{1} c_{0}(t)+\frac{1}{2} \sigma_{1}^{2}(t)+c_{1}(t)+\left[-a_{2}(t) y^{2}\right. \\
& +\left(a_{2}(t)+r_{2}(t)-d_{2} c_{0}(t)+\beta(t)\right) y-r_{2}(t) \\
& \left.+d_{2} c_{0}(t)+\frac{1}{2} \sigma_{2}^{2}(t)+c_{2}(t)\right] \leq K_{1},
\end{aligned}
$$

where

$$
K_{1}=\max _{0 \leq t<+\infty} \sum_{i=1}^{2} \frac{4 a_{i}(t)\left[-r_{i}(t)+d_{i} c_{0}(t)+(1 / 2) \sigma_{i}^{2}(t)+c_{i}(t)\right]+\left[a_{i}(t)+r_{i}(t)-d_{i} c_{0}(t)+\beta(t)\right]^{2}}{4 a_{i}(t)} .
$$

So

$$
\begin{aligned}
& d V(x, y) \leq K_{1} d t-(x-1) \sigma_{1}(t) d B_{1}(t)-(y-1) \\
& \quad \cdot \sigma_{2}(t) d B_{2}(t) \\
& \quad+\int_{\mathbb{V}}\left[x\left(t^{-}\right) \gamma_{1}(t, u)-\ln \left(1+\gamma_{1}(t, u)\right)\right] \widetilde{N}(d t, d u) \\
& \quad+\int_{\mathbb{V}}\left[y\left(t^{-}\right) \gamma_{2}(t, u)-\ln \left(1+\gamma_{2}(t, u)\right)\right] \widetilde{N}(d t, d u) .
\end{aligned}
$$

Integrating (18) from 0 to $\tau_{k} \wedge T$ and taking the expectations for both sides result in

$$
\mathbb{E}\left[V\left(x\left(\tau_{k} \wedge T\right), y\left(\tau_{k} \wedge T\right)\right)\right] \leq V\left(x_{0}, y_{0}\right)+K_{1} T .
$$

Let $\Omega_{k}=\left\{\tau_{k} \leq T\right\}$ for $k \geq k_{1}$; we have $P\left(\Omega_{k}\right) \geq \varepsilon$. Then

$$
\begin{aligned}
& V\left(x\left(\tau_{k} \wedge T, \omega\right), y\left(\tau_{k} \wedge T, \omega\right)\right) \\
& \quad \geq[k-1-\ln k] \wedge\left[\frac{1}{k}-1+\ln k\right] .
\end{aligned}
$$

It is inferred from (19) and (20) that

$$
\begin{aligned}
V\left(x_{0}, y_{0}\right)+K_{1} T & \geq \mathbb{E}\left[1_{\Omega} V\left(x\left(\tau_{k} \wedge T\right), y\left(\tau_{k} \wedge T\right)\right)\right] \\
& \geq \varepsilon[k-1-\ln k] \wedge\left[\frac{1}{k}-1+\ln k\right],
\end{aligned}
$$

where $1_{\Omega}$ is the indicator function of $\Omega_{k}$. Let $k \rightarrow+\infty$; we have that

$$
+\infty>V\left(x_{0}, y_{0}\right)+K_{1} T \geq+\infty
$$

is a contradiction; then we have $\tau_{\infty}=\infty$.

This completes the proof of Theorem 4 .

\section{Main Results}

3.1. Extinction. For convenience, we prepare the following lemma.

Lemma 5. Suppose that $\eta(t) \in C\left(\Omega \times[0, \infty), \mathbb{R}_{+}\right)$and let Assumption 3 hold.

(I) If there exist two positive constants $T$ and $\delta_{0}$ such that

$$
\begin{aligned}
\ln \eta(t) \leq & \int_{0}^{t} \delta(s) d s-\delta_{0} \int_{0}^{t} \eta(s) d s+\alpha B(t) \\
& +\sum_{i=1}^{2} \delta_{i} \int_{0}^{t} \int_{\mathbb{V}} \ln \left(1+\gamma_{i}(u)\right) \widetilde{N}(d s, d u)
\end{aligned}
$$

a.s.

for all $t \geq T$, where $\alpha, \delta_{1}$, and $\delta_{2}$ are constants, then

$$
\begin{aligned}
\langle\eta\rangle^{*} & \leq \frac{\langle\delta\rangle^{*}}{\delta_{0}} \quad \text { a.s., if }\langle\delta\rangle^{*} \geq 0 ; \\
\lim _{t \rightarrow \infty} \eta(t) & =0 \quad \text { a.s., if }\langle\delta\rangle^{*}<0 .
\end{aligned}
$$

(II) If there exist two positive constants $T$ and $\delta_{0}$ such that

$$
\begin{aligned}
\ln \eta(t) \geq & \int_{0}^{t} \delta(s) d s-\delta_{0} \int_{0}^{t} \eta(s) d s+\alpha B(t) \\
& +\sum_{i=1}^{2} \delta_{i} \int_{0}^{t} \int_{\mathbb{Y}} \ln \left(1+\gamma_{i}(u)\right) \widetilde{N}(d s, d u) \quad \text { a.s. }
\end{aligned}
$$

for all $t \geq T$, then $\langle\eta\rangle_{*} \geq\langle\delta\rangle_{*} / \delta_{0}$ a.s. provided that $\langle\delta\rangle_{*} \geq 0$. 
Lemma 5 is proved in Appendix by using a similar method in [13].

Now, we will prove the extinction of system (4).

Define

$$
\begin{aligned}
b_{1}(t)= & r_{1}(t)-\frac{1}{2} \sigma_{1}^{2}(t) \\
& +\int_{\mathbb{Y}}\left(\ln \left(1+\gamma_{1}(t, u)\right)-\gamma_{1}(t, u)\right) \nu(d u), \\
b_{2}(t)= & r_{2}(t)-\frac{1}{2} \sigma_{2}^{2}(t) \\
& +\int_{\mathbb{Y}}\left(\ln \left(1+\gamma_{2}(t, u)\right)-\gamma_{2}(t, u)\right) \nu(d u), \\
\overline{c_{0}}= & \frac{k u}{h(g+m) \tau} .
\end{aligned}
$$

Theorem 6. If

$$
\begin{aligned}
& \left\langle b_{1}\right\rangle_{*}<d_{1} \overline{c_{0}} \\
& \left\langle b_{2}\right\rangle_{*}<d_{2} \overline{c_{0}}
\end{aligned}
$$

then

$$
\begin{aligned}
& \lim _{t \rightarrow \infty} x(t)=0, \\
& \lim _{t \rightarrow \infty} y(t)=0
\end{aligned}
$$

a.s.

Proof. By Assumption 3 and the strong law of large numbers for local martingales, one has

$$
\begin{aligned}
& \lim _{t \rightarrow+\infty} \frac{1}{t} \int_{0}^{t} \int_{\mathbb{V}} \ln \left(1+\gamma_{i}(s, u)\right) \widetilde{N}(d s, d u)=0, \\
& \lim _{t \rightarrow+\infty} \frac{\int_{0}^{t} \sigma_{i}(s) d B_{i}(s)}{t}=0 \\
& \text { a.s., } i=1,2 .
\end{aligned}
$$

Define

$$
\begin{aligned}
& V_{1}(x)=\ln x(t), \\
& V_{2}(x)=\ln y(t) .
\end{aligned}
$$

Applying Itô's formula yields

$$
\begin{aligned}
d \ln x & (t) \\
= & \left(b_{1}(t)-d_{1} c_{0}(t)-a_{1}(t) x(t)-\beta(t) y(t)\right) d t \\
& -\sigma_{1}(t) d B_{1}(t) \\
& +\int_{\mathbb{V}} \ln \left(1+\gamma_{1}(t, u)\right) \widetilde{N}(d t, d u),
\end{aligned}
$$

$d \ln y(t)$

$$
\begin{aligned}
= & \left(b_{2}(t)-d_{2} c_{0}(t)-a_{2}(t) y(t)+\beta(t) x(t)\right) d t \\
& -\sigma_{2}(t) d B_{2}(t) \\
& +\int_{\mathbb{Y}} \ln \left(1+\gamma_{2}(t, u)\right) \widetilde{N}(d t, d u) .
\end{aligned}
$$

Integrating both sides of (32) and (33) from 0 to $t$, respectively, we have

$$
\begin{aligned}
& \ln x(t)-\ln x(0) \\
& =\int_{0}^{t}\left(b_{1}(s)-d_{1} c_{0}(s)-a_{1}(s) x(s)-\beta(s) y(s)\right) d t \\
& \quad-\int_{0}^{t} \sigma_{1}(s) d B_{1}(s) \\
& \quad+\int_{0}^{t} \int_{\mathbb{V}} \ln \left(1+\gamma_{1}(t, u)\right) \widetilde{N}(d t, d u), \\
& \ln y(t)-\ln y(0) \\
& =\int_{0}^{t}\left(b_{2}(s)-d_{2} c_{0}(s)-a_{2}(s) y(s)+\beta(s) x(s)\right) d s \\
& \quad-\int_{0}^{t} \sigma_{2}(s) d B_{2}(s) \\
& \quad+\int_{0}^{t} \int_{\mathbb{Y}} \ln \left(1+\gamma_{2}(t, u)\right) \widetilde{N}(d t, d u) .
\end{aligned}
$$

From (34), we can obtain that

$$
\begin{aligned}
& \frac{\ln x(t)-\ln x(0)}{t} \\
& \leq \frac{\int_{0}^{t} b_{1}(s) d s}{t}-d_{1} \frac{\int_{0}^{t} c_{0}(s) d s}{t}-\frac{\int_{0}^{t} \sigma_{1}(s) d B_{1}(s)}{t} \\
& \quad+\frac{\int_{0}^{t} \int_{\mathbb{V}} \ln \left(1+\gamma_{1}(t, u)\right) \widetilde{N}(d t, d u)}{t} .
\end{aligned}
$$

Taking the limit superior results in

$$
\left[\frac{\ln x(t)}{t}\right]^{*} \leq \limsup _{t \rightarrow \infty}\left[\frac{\int_{0}^{t} b_{1}(s) d s}{t}-d_{1} \frac{\int_{0}^{t} c_{0}(s) d s}{t}\right]
$$

From (27) we can know that $[\ln x(t) / t]^{*}<0$. Thus we have

$$
\lim _{t \rightarrow \infty} x(t)=0 \quad \text { a.s. }
$$


Applying (35) leads to

$$
\begin{aligned}
& \frac{\ln y(t)-\ln y(0)}{t} \\
& \leq \frac{\int_{0}^{t} b_{2}(s) d s}{t}+\frac{\int_{0}^{t} \beta(s) x(s) d s}{t}-d_{2} \frac{\int_{0}^{t} c_{0}(s) d s}{t} \\
& \quad-\frac{\int_{0}^{t} \sigma_{2}(s) d B_{2}(s)}{t} \\
& \quad+\frac{\int_{0}^{t} \int_{\mathbb{V}} \ln \left(1+\gamma_{2}(t, u)\right) \widetilde{N}(d t, d u)}{t} .
\end{aligned}
$$

Taking the limit superior, together with (28) and (38), we can see that

$$
\left[\frac{\ln y(t)}{t}\right]^{*}<0 \quad \text { a.s. }
$$

Therefore,

$$
\lim _{t \rightarrow \infty} y(t)=0 \quad \text { a.s. }
$$

This completes the proof of Theorem 6 .

For simplicity, we define

$$
R^{*}=\frac{\left\langle b_{2}\right\rangle^{*}+\beta^{M} / a_{1}^{L} \cdot\left\langle b_{1}\right\rangle^{*}}{\left(d_{2}+d_{1}\left(\beta^{M} / a_{1}^{L}\right)\right) \overline{c_{0}}} .
$$

Theorem 7. If $\left\langle b_{1}\right\rangle_{*}>d_{1} \overline{c_{0}}$ and $R^{*}<1$, then

$$
\begin{aligned}
\frac{\left\langle b_{1}\right\rangle_{*}-d_{1} \overline{c_{0}}}{a_{1}^{M}} & \leq\langle x(t)\rangle_{*} \leq\langle x(t)\rangle^{*} \leq \frac{\left\langle b_{1}\right\rangle^{*}-d_{1} \overline{c_{0}}}{a_{1}^{L}}, \\
\lim _{t \rightarrow \infty} y(t) & =0
\end{aligned}
$$

a.s.

Proof. According to (34), we can obtain that

$$
\begin{aligned}
& \ln x(t)-\ln x(0) \\
& \leq \int_{0}^{t} b_{1}(s) d s-d_{1} \int_{0}^{t} c_{0}(s) d s-\int_{0}^{t} a_{1}(s) x(s) d s \\
& \quad-\int_{0}^{t} \sigma_{1}(s) d B_{1}(s) \\
& \quad+\int_{0}^{t} \int_{\mathbb{Y}} \ln \left(1+\gamma_{1}(t, u)\right) \widetilde{N}(d t, d u) \\
& \leq \int_{0}^{t} b_{1}(s) d s-d_{1} \int_{0}^{t} c_{0}(s) d s-a_{1}^{L} \int_{0}^{t} x(s) d s \\
& \quad-\int_{0}^{t} \sigma_{1}(s) d B_{1}(s) \\
& \quad+\int_{0}^{t} \int_{\mathbb{Y}} \ln \left(1+\gamma_{1}(t, u)\right) \widetilde{N}(d t, d u) .
\end{aligned}
$$

It follows from Lemma 5 that

$$
\langle x(t)\rangle^{*} \leq \frac{\left\langle b_{1}\right\rangle^{*}-d_{1} \overline{c_{0}}}{a_{1}^{L}} \quad \text { a.s. }
$$

By (35), we have

$$
\begin{aligned}
& \ln y(t)-\ln y(0) \leq \int_{0}^{t} b_{2}(s) d s+\int_{0}^{t} \beta(s) x(s) d s \\
& -d_{2} \int_{0}^{t} c_{0}(s) d s-\int_{0}^{t} a_{2}(s) y(s) d s \\
& \quad-\int_{0}^{t} \sigma_{2}(s) d B_{2}(s) \\
& +\int_{0}^{t} \int_{\mathbb{Y}} \ln \left(1+\gamma_{2}(t, u)\right) \widetilde{N}(d t, d u) \\
& \leq \int_{0}^{t} \frac{\int_{0}(s) d s}{t}+\beta^{M} \frac{\left\langle b_{1}\right\rangle^{*}-d_{1} \overline{c_{0}}}{a_{1}^{L}} \\
& \left.\quad-d_{2} \frac{\int_{0}^{t} c_{0}(s) d s}{t}\right) t-\int_{0}^{t} \sigma_{2}(s) d B_{2}(s) \\
& +\int_{0}^{t} \int_{\mathbb{Y}} \ln \left(1+\gamma_{2}(t, u)\right) \widetilde{N}(d t, d u),
\end{aligned}
$$

and then

$$
\begin{aligned}
& y(t) \leq y(0) \exp \left\{\left(\frac{\int_{0}^{t} b_{2}(t) d t}{t}+\beta^{M} \frac{\left\langle b_{1}\right\rangle^{*}-d_{1} \overline{c_{0}}}{a_{1}^{L}}\right.\right. \\
& \left.-d_{2} \frac{\int_{0}^{t} c_{0}(s) d s}{t}\right) t-\int_{0}^{t} \sigma_{2}(t) d B_{2}(t) \\
& \left.+\int_{0}^{t} \int_{\mathbb{V}} \ln \left(1+\gamma_{2}(t, u)\right) \tilde{N}(d t, d u)\right\} .
\end{aligned}
$$

Since $R^{*}<1$, then we know that

$$
\lim _{t \rightarrow \infty} y(t)=0 \text { a.s., }
$$

which combined with (34) and the property of the limit superior shows that, for any $\varepsilon>0$, there exists a random number $T>0$ for $t>T$ such that

$$
\begin{aligned}
\ln \frac{x(t)}{x(0)} \geq & \int_{0}^{t}\left(b_{1}(s)-d_{1} c_{0}(s)\right) d s-\beta^{M} \varepsilon \\
& -a_{1}^{M} \int_{0}^{t} x(t) d t-\int_{0}^{t} \sigma_{1}(t) d B_{1}(t) \\
& +\int_{0}^{t} \int_{\mathbb{V}} \ln \left(1+\gamma_{1}(t, u)\right) \widetilde{N}(d t, d u) .
\end{aligned}
$$

By Lemma 5, we have that

$$
\langle x(t)\rangle_{*} \geq \frac{\left\langle b_{1}\right\rangle_{*}-d_{1} \overline{c_{0}}}{a_{1}^{M}} \quad \text { a.s. }
$$

This completes the proof of Theorem 7. 
3.2. Permanence in Mean. In this section, we need to show the permanence in mean. Note that $\left\langle b_{1}\right\rangle_{*}\left\langle d_{1} \overline{c_{0}}\right.$ implies that productiveness of the prey is less than its death loss rate; then the prey can go extinct. Naturally, we assume $\left\langle b_{1}\right\rangle_{*}>d_{1} \overline{c_{0}}$ in the rest of this paper.

Define

$$
\begin{aligned}
& \Delta_{1}=\beta^{L}\left\langle b_{1}\right\rangle_{*}+a_{1}^{M}\left\langle b_{2}\right\rangle_{*}-\left(\beta^{L} d_{1}+a_{1}^{M} d_{2}\right) \overline{c_{0}}, \\
& \Delta_{2}=\beta^{L} \beta^{M}+a_{1}^{M} a_{2}^{M}, \\
& \Delta_{3}=\left\langle b_{1}\right\rangle_{*}-d_{1} \overline{c_{0}}-\beta^{L} \frac{\Delta_{1}}{\Delta_{2}}, \\
& \Delta_{4}=\left\langle b_{1}\right\rangle_{*}-d_{1} \overline{c_{0}}-\beta^{M} \frac{\Delta_{5}}{a_{2}^{L}} \\
& \Delta_{5}=\left\langle b_{2}\right\rangle^{*}-d_{2} \overline{c_{0}}+\beta^{M} \frac{\Delta_{3}}{a_{1}^{L}} \\
& R_{*}=\frac{\left\langle b_{2}\right\rangle_{*}+\beta^{L} / a_{1}^{M} \cdot\left\langle b_{1}\right\rangle_{*}}{\left(d_{2}+d_{1}\left(\beta^{L} / a_{1}^{M}\right)\right) \overline{c_{0}}} .
\end{aligned}
$$

Theorem 8. If

$$
\begin{aligned}
\left\langle b_{2}\right\rangle^{*} & >d_{2} \overline{c_{0}}, \\
R_{*} & >1, \\
\Delta_{3} & >0, \\
\Delta_{4} & >0,
\end{aligned}
$$

then

$$
\begin{aligned}
& \frac{\Delta_{4}}{a_{1}^{M}} \leq\langle x(t)\rangle_{*} \leq\langle x(t)\rangle^{*} \leq \frac{\Delta_{3}}{a_{1}^{L}}, \\
& \frac{\Delta_{1}}{\Delta_{2}} \leq\langle y(t)\rangle_{*} \leq\langle y(t)\rangle^{*} \leq \frac{\Delta_{5}}{a_{2}^{L}} .
\end{aligned}
$$

Proof. Define $u(t)$ such that $x(t) \leq u(t)$, where $u(t)$ satisfies

$$
\begin{aligned}
d u(t)= & u(t)\left(r_{1}(t)-a_{1}(t) u(t)\right) d t \\
& -\sigma_{1}(t) u(t) d B_{1}(t) \\
& +\int_{\mathbb{Y}} u\left(t^{-}\right) \gamma_{1}(t, u) \widetilde{N}(d t, d u)
\end{aligned}
$$

In [9], we know that

$$
\lim _{t \rightarrow \infty} \frac{\ln u(t)}{t}=0, \quad \text { a.s. }
$$

It can be inferred from comparison theorem that

$$
\left(\frac{\ln x(t)}{t}\right)^{*} \leq \lim _{t \rightarrow \infty} \frac{\ln u(t)}{t}=0
$$

From (34) and (35), we know that

$$
\begin{aligned}
& \frac{\ln x(t)-\ln x(0)}{t} \\
& =\frac{\int_{0}^{t} b_{1}(s) d s}{t}-\frac{\int_{0}^{t} d_{1} c_{0}(s) d s}{t}-\frac{\int_{0}^{t} a_{1}(s) x(s) d s}{t} \\
& \quad-\frac{\int_{0}^{t} \beta(s) y(s) d s}{t}-\frac{\int_{0}^{t} \sigma_{1}(s) d B_{1}(s)}{t} \\
& \quad+\frac{\int_{0}^{t} \int_{\mathbb{Y}} \ln \left(1+\gamma_{1}(t, u)\right) \widetilde{N}(d t, d u)}{t}, \\
& \frac{\ln y(t)-\ln y(0)}{t} \\
& \quad \frac{\int_{0}^{t} b_{2}(s) d s}{t}-\frac{\int_{0}^{t} d_{2} c_{0}(s) d s}{t}-\frac{\int_{0}^{t} a_{2}(s) y(s) d s}{t} \\
& +\frac{\int_{0}^{t} \beta(s) x(s) d s}{t}-\frac{\int_{0}^{t} \sigma_{2}(s) d B_{2}(s)}{t}
\end{aligned}
$$

By $\beta_{L} \times(57)+a_{1}^{M} \times(58)$, we obtain that

$$
\begin{gathered}
\beta^{L} \times \frac{\ln x(t)-\ln x(0)}{t}+a_{1}^{M} \times \frac{\ln y(t)-\ln y(0)}{t} \\
\geq \Delta_{1}-\Delta_{2}\langle y(t)\rangle-\beta_{L} \frac{\int_{0}^{t} \sigma_{1}(s) d B_{1}(s)}{t} \\
\quad+\beta_{L} \frac{\int_{0}^{t} \int_{\mathbb{Y}} \ln \left(1+\gamma_{1}(t, u)\right) \widetilde{N}(d t, d u)}{t} \\
\quad-a_{1}^{M} \frac{\int_{0}^{t} \sigma_{2}(s) d B_{2}(s)}{t} \\
+a_{1}^{M} \frac{\int_{0}^{t} \int_{\mathbb{V}} \ln \left(1+\gamma_{2}(t, u)\right) \widetilde{N}(d t, d u)}{t}
\end{gathered}
$$

When $R_{*}>1$ is used in (59), applying Lemma 5 and (56) leads to

$$
\langle y(t)\rangle_{*} \geq \frac{\Delta_{1}}{\Delta_{2}} .
$$

According to (57) and (60), we can have

$$
\begin{aligned}
& \frac{\ln x(t)-\ln x(0)}{t} \\
& \leq\left\langle b_{1}\right\rangle^{*}-d_{1} \overline{c_{0}}-\beta^{L} \frac{\Delta_{1}}{\Delta_{2}}-a_{1}^{L}\langle x(t)\rangle \\
& \quad-\frac{\int_{0}^{t} \sigma_{1}(s) d B_{1}(s)}{t} \\
& +\frac{\int_{0}^{t} \int_{\mathbb{V}} \ln \left(1+\gamma_{1}(t, u)\right) \widetilde{N}(d t, d u)}{t} .
\end{aligned}
$$


From the conditions of Theorem 8, we know that $\Delta_{3}>0$ and applying Lemma 5 results in

$$
\langle x(t)\rangle^{*} \leq \frac{\Delta_{3}}{a_{1}^{L}} .
$$

By (58) and (62), we obtain that

$$
\begin{aligned}
& \frac{\ln y(t)-\ln y(0)}{t} \\
& \leq\left\langle b_{2}\right\rangle^{*}-d_{2} \overline{c_{0}}+\beta^{M} \frac{\Delta_{3}}{a_{1}^{L}}-a_{2}^{L}\langle y(t)\rangle \\
& \quad-\frac{\int_{0}^{t} \sigma_{1}(s) d B_{1}(s)}{t} \\
& +\frac{\int_{0}^{t} \int_{\mathbb{V}} \ln \left(1+\gamma_{1}(t, u)\right) \widetilde{N}(d t, d u)}{t} .
\end{aligned}
$$

From the conditions of Theorem 8, we see that $\Delta_{5}>0$ and applying Lemma 5 leads to

$$
\langle y(t)\rangle^{*} \leq \frac{\Delta_{5}}{a_{2}^{L}} .
$$

By (58) and (64), we can have

$$
\begin{aligned}
& \frac{\ln x(t)-\ln x(0)}{t} \\
& \geq\left\langle b_{1}\right\rangle_{*}-d_{1} \overline{c_{0}}-\beta^{M} \frac{\Delta_{5}}{a_{2}^{L}}-a_{1}^{M}\langle x(t)\rangle \\
& \quad-\frac{\int_{0}^{t} \sigma_{1}(s) d B_{1}(s)}{t} \\
& \quad+\frac{\int_{0}^{t} \int_{\mathbb{V}} \ln \left(1+\gamma_{1}(t, u)\right) \widetilde{N}(d t, d u)}{t} .
\end{aligned}
$$

According to the conditions of Theorem 8, we see that $\Delta_{4}>0$ and applying Lemma 5 yields

$$
\langle x(t)\rangle_{*} \geq \frac{\Delta_{4}}{a_{1}^{M}} .
$$

This completes the proof of Theorem 8 .

3.3. Stochastic Permanence. For the sake of proving stochastic permanence, we should examine the $p$ th moment boundedness firstly.

Define

$$
\begin{aligned}
\lambda_{1} & =\max _{t \in[0,+\infty)}\left\{p r_{1}(t)-p d_{1} c_{0}(t)+\frac{p(p-1)}{2} \sigma_{1}^{2}(t)\right. \\
& \left.+c_{1}(t)\right\},
\end{aligned}
$$

$$
\begin{aligned}
\lambda_{1}^{\prime} & =\max _{t \in[0,+\infty)}\left\{p r_{2}(t)-p d_{2} c_{0}(t)+\frac{p(p-1)}{2} \sigma_{2}^{2}(t)\right. \\
& \left.+c_{2}(t)\right\} \\
\lambda_{1}^{\prime \prime} & =\max \left\{\lambda_{1}, \lambda_{1}^{\prime}\right\} \\
\lambda_{2} & =\min _{t \in[0,+\infty)}\left\{a_{1}(t) p-\frac{p \beta(t)}{p+1}\right\}, \\
\lambda_{2}^{\prime} & =\min _{t \in[0,+\infty)}\left\{a_{2}(t) p-\frac{p^{2} \beta(t)}{p+1}\right\}, \\
\lambda_{2}^{\prime \prime} & =\min \left\{\lambda_{2}, \lambda_{2}^{\prime}\right\} .
\end{aligned}
$$

Theorem 9. If there exists a constant $p$ such that

$$
\begin{array}{r}
\int_{\mathbb{V}}\left(\left(1+\gamma_{i}(t, u)\right)^{p}-1-p \gamma_{i}(t, u)\right) v(d u)<\infty, \\
\lambda_{1}^{\prime \prime}>0, \\
\lambda_{2}^{\prime \prime}>0,
\end{array}
$$

then

$$
\limsup _{t \rightarrow \infty} \mathbb{E}\left[\left(x^{2}(t)+y^{2}(t)\right)^{p / 2}\right] \leq K(p) \quad \text { a.s. }
$$

Proof. Define

$$
V(x, y)=x^{p}(t)+y^{p}(t) .
$$

Applying Itô's formula and (68) yields

$$
\begin{aligned}
d V(x, y) & \\
= & L V d t-p \sigma_{1}(t) x^{p}(t) d B_{1}(t) \\
& -p \sigma_{2}(t) y^{p}(t) d B_{2}(t) \\
& +\int_{\mathbb{V}} x^{p}\left(t^{-}\right)\left(\left(1+\gamma_{1}(t, u)\right)^{p}-1\right) \widetilde{N}(d t, d u) \\
& +\int_{\mathbb{V}} y^{p}\left(t^{-}\right)\left(\left(1+\gamma_{2}(t, u)\right)^{p}-1\right) \widetilde{N}(d t, d u),
\end{aligned}
$$

where

$$
\begin{aligned}
L V & =p x^{p}(t)\left(r_{1}(t)-d_{1} c_{0}(t)-a_{1}(t) x(t)-\beta(t)\right. \\
\cdot & y(t))+p y^{p}(t)\left(r_{2}(t)-d_{2} c_{0}(t)-a_{2}(t) x(t)\right. \\
& +\beta(t) x(t))+\frac{p(p-1)}{2}\left(\sigma_{1}^{2}(t) x^{p}(t)+\sigma_{2}^{2}(t)\right. \\
& \left.\cdot y^{p}(t)\right)+\int_{\mathbb{V}} x^{p}\left(t^{-}\right) \\
& \cdot\left(\left(1+\gamma_{1}(t, u)\right)^{p}-1-p \gamma_{1}(t, u)\right) \nu(d u)
\end{aligned}
$$




$$
\begin{aligned}
& +\int_{\mathbb{Y}} y^{p}\left(t^{-}\right)\left(\left(1+\gamma_{1}(t, u)\right)^{p}-1-p \gamma_{1}(t, u)\right) \\
& \cdot v(d u) \leq\left(p r_{1}(t)-p d_{1} c_{0}(t)+\frac{p(p-1)}{2} \sigma_{1}^{2}(t)\right. \\
& \left.+c_{1}(t)\right) x^{p}(t)+\left(p r_{2}(t)-p d_{2} c_{0}(t)+\frac{p(p-1)}{2}\right. \\
& \left.\cdot \sigma_{2}^{2}(t)+c_{2}(t)\right) y^{p}(t)-a_{1}(t) p x^{p+1}(t)-\beta(t) \\
& \cdot p x^{p}(t) y(t)-a_{2}(t) p y^{p+1}(t)+p \beta(t) x(t) y^{p}(t) \\
& \leq\left(p r_{1}(t)-p d_{1} c_{0}(t)+\frac{p(p-1)}{2} \sigma_{1}^{2}(t)+c_{1}(t)\right) \\
& \cdot x^{p}(t)-\left(a_{1}(t) p-\frac{p \beta(t)}{p+1}\right) x^{p+1}(t)+\left(p r_{2}(t)\right. \\
& \left.-p d_{2} c_{0}(t)+\frac{p(p-1)}{2} \sigma_{2}^{2}(t)+c_{2}(t)\right) y^{p}(t) \\
& -\left(a_{2}(t) p-\frac{p^{2} \beta(t)}{p+1}\right) y^{p+1}(t), \\
& \left(\begin{array}{c}
p \\
\end{array}(t)\right.
\end{aligned}
$$

where $c_{i}(t)(i=1,2)$ is a bounded continuous function.

Integrating (72) from 0 to $t$ and taking the expectations for both sides yield

$$
\mathbb{E}[V(x, y)]=V(x(0), y(0))+\mathbb{E}\left[\int_{0}^{t} L V d t\right],
$$

and then

$$
\begin{aligned}
\frac{d \mathbb{E}[}{d t} & V(x, y)] \\
\leq & \lambda_{1} \mathbb{E}\left[x^{p}\right]-\lambda_{2} \mathbb{E}\left[x^{p+1}\right]+\lambda_{1}^{\prime} \mathbb{E}\left[y^{p}\right] \\
& -\lambda_{2}^{\prime} \mathbb{E}\left[y^{p+1}\right] \\
\leq & \lambda_{1}^{\prime \prime} \mathbb{E}\left[x^{p}+y^{p}\right]-\lambda_{2}^{\prime \prime} \mathbb{E}\left[x^{p+1}+y^{p+1}\right] \\
\leq & \lambda_{1}^{\prime \prime} \mathbb{E}\left[x^{p}+y^{p}\right]-\lambda_{2}^{\prime \prime} \cdot 2^{-1 / p}\left(\mathbb{E}\left[x^{p}+y^{p}\right]\right)^{(p+1) / p} \\
= & \mathbb{E}\left[x^{p}+y^{p}\right]\left(\lambda_{1}^{\prime \prime}-\lambda_{2}^{\prime \prime} \cdot 2^{-1 / p}\left(\mathbb{E}\left[x^{p}+y^{p}\right]\right)^{1 / p}\right) .
\end{aligned}
$$

We know that the auxiliary equation

$$
\dot{z}(t)=z(t)\left[\lambda_{1}-\lambda_{2}(z(t))^{1 / p}\right]
$$

has a globally asymptotically stable positive equilibrium $\bar{z}=$ $\left(\lambda_{1} / \lambda_{2}\right)^{p}$. Let $z(t)$ be the solution of (76) with $z(0)=$ $\mathbb{E}\left[(x(0))^{p}+(y(0))^{p}\right]$, and according to the comparison theorem we have $\mathbb{E}\left[x^{p}+y^{p}\right] \leq z(t), t \geq 0$. Hence, we obtain

$$
\limsup _{t \rightarrow+\infty} \mathbb{E}[V(x, y)] \leq 2^{1 / p} \cdot \frac{\lambda_{1}^{\prime \prime}}{\lambda_{2}^{\prime \prime}}:=K_{1}(p) \quad \text { a.s. }
$$

We find that

$$
\left(x^{2}+y^{2}\right)^{p / 2} \leq 2^{p / 2} \cdot \max \left\{x^{p}, y^{p}\right\} \leq 2^{p / 2} \cdot V(x, y),
$$

so we have

$$
\limsup _{t \rightarrow+\infty} \mathbb{E}\left[\left(x^{2}+y^{2}\right)^{p / 2}\right] \leq \sqrt{2} \cdot \frac{\lambda_{1}^{\prime \prime}}{\lambda_{2}^{\prime \prime}}:=K(p) \quad \text { a.s. }
$$

This completes the proof of Theorem 9 .

$$
\begin{aligned}
& r(t)=\min \left\{r_{1}(t)-d_{1} c_{0}(t), r_{2}(t)-d_{2} c_{0}(t)\right\}, \\
& \sigma(t)=\max \left\{\sigma_{1}(t), \sigma_{2}(t)\right\} \text {, } \\
& H(t)=r(t)-\sigma^{2}(t)-\sum_{i=1}^{2} \int_{\mathbb{Y}} \frac{\gamma_{i}^{2}}{1+\gamma_{i}} v(d u) .
\end{aligned}
$$

Theorem 10. If

$$
\langle H(t)\rangle_{*}>0,
$$

then system (4) is stochastically permanent.

Proof. Define

$$
V(x, y)=\frac{1}{x(t)+y(t)} .
$$

By applying Itô's formula, we have

$$
\begin{aligned}
& d(V(x, y))=L V d t+\frac{1}{(x+y)^{2}}\left(\sigma_{1}(t) x(t) d B_{1}(t)\right. \\
& \left.\quad+\sigma_{2}(t) y(t) d B_{2}(t)\right) \\
& +\int_{\mathbb{V}}\left(\frac{1}{x\left(t^{-}\right)+y\left(t^{-}\right)+x\left(t^{-}\right) \gamma_{1}(t, u)+y\left(t^{-}\right) \gamma_{2}(t, u)}\right. \\
& \left.\quad-\frac{1}{x\left(t^{-}\right)+y\left(t^{-}\right)}\right) \widetilde{N}(d t, d u),
\end{aligned}
$$

where

$$
\begin{aligned}
L V & =-\frac{1}{(x+y)^{2}}\left(x(t)\left(r_{1}(t)-d_{1} c_{0}(t)\right)-a_{1}(t) x^{2}(t)\right. \\
& \left.+\left(r_{2}(t)-d_{2} c_{0}(t)\right) y(t)-a_{2}(t) y^{2}(t)\right) \\
& +\frac{\sigma_{1}^{2}(t) x^{2}(t)+\sigma_{2}^{2}(t) y^{2}(t)+2 \sigma_{1}(t) \sigma_{2}(t) x(t) y(t)}{(x(t)+y(t))^{3}} \\
& +\int_{\mathbb{Y}}\left(\frac{1}{x+y+x \gamma_{1}(t, u)+y \gamma_{2}(t, u)}-\frac{1}{x+y}\right. \\
& \left.+\frac{1}{(x+y)^{2}}\left(x \gamma_{1}(t, u)+y \gamma_{2}(t, u)\right)\right) \nu(d u) \\
& \leq-\frac{1}{x(t)+y(t)}\left(r(t)-\sigma^{2}(t)\right. \\
& \left.-\sum_{i=1}^{2} \int_{\mathbb{Y}} \frac{\gamma_{i}^{2}(t, u)}{1+\gamma_{i}(t, u)} v(d u)\right)+a(t)=-V \cdot H(t) \\
& +a(t),
\end{aligned}
$$

where $a(t)=\max \left\{a_{1}(t), a_{2}(t)\right\}$. 
Integrating (83) from 0 to $t$ and taking the expectation on both sides yield

$$
\mathbb{E}[V(t)]=V(0)+\mathbb{E}\left[\int_{0}^{t} L V(s) d s\right] .
$$

Therefore,

$$
\begin{aligned}
d \mathbb{E}[V(t)] & =\mathbb{E}[L V(t)] d t \\
& \leq(-H(t) \mathbb{E}[V(t)]+a(t)) d t .
\end{aligned}
$$

By the variation of constants method, we can derive that

$$
\begin{aligned}
\mathbb{E}[V(t)] \leq & \int_{0}^{t} a(s) \exp \left(-\int_{s}^{t} H(\tau) d \tau\right) d s \\
& +V(0) \exp \left(-\int_{0}^{t} H(s) d s\right),
\end{aligned}
$$

which together with (81) results in

$$
\mathbb{E}[V(x, y)] \leq K,
$$

where $K$ is a positive constant. Then, for any given $\varepsilon>0$ and constant $\delta(\varepsilon)=\varepsilon / K$, according to the Chebyshev inequality, we can know that

$$
\begin{aligned}
P\left\{\frac{1}{V(x, y)}<\delta\right\} & =P\left\{V(x, y)>\frac{1}{\delta}\right\} \\
& \leq \frac{\mathbb{E}[V(x, y)]}{1 / \delta}=\delta \mathbb{E}[V(x, y)] \\
& \leq \varepsilon
\end{aligned}
$$

and then

$$
P\left\{\frac{1}{V(x, y)} \geq \delta\right\} \geq 1-\varepsilon
$$

Therefore,

$$
\liminf _{t \rightarrow \infty} P\{(x(t)+y(t)) \geq \delta\} \geq 1-\varepsilon \quad \text { a.s. }
$$

Then, we have

$$
\liminf _{t \rightarrow \infty}\left\{\left(\sqrt{x^{2}(t)+y^{2}(t)}\right) \geq \frac{\sqrt{2}}{2} \delta\right\} \geq 1-\varepsilon \quad \text { a.s. }
$$

Using Chebyshev inequality and Theorem 9, we can obtain

$$
\limsup _{t \rightarrow \infty} P\left\{\left(\sqrt{x^{2}(t)+y^{2}(t)}\right) \leq \chi\right\} \geq 1-\varepsilon \quad \text { a.s., }
$$

where $\chi$ is a constant. This completes the proof of Theorem 10 .

\section{Simulations and Conclusions}

In this section, we show some numerical examples, which will demonstrate our results.
Choose the parameters in (4) as follows:

$$
\begin{aligned}
r_{1}(t) & =1.8, \\
r_{2}(t) & =1.3, \\
d_{1} & =0.35, \\
d_{2} & =0.4, \\
a_{1}(t) & =0.3, \\
a_{2}(t) & =0.4, \\
\beta(t) & =0.1, \\
k & =0.5, \\
g & =0.2, \\
h & =0.2, \\
m & =0.3, \\
u & =1, \\
\tau & =1 .
\end{aligned}
$$

In Figure 1, we choose $\sigma_{1}(t)=0.3, \sigma_{2}(t)=0.4$ and the values of $\gamma_{1}(t, u)$ and $\gamma_{2}(t, u)$ are different between (a) and (b).

In Figure 1(a), we choose $\gamma_{1}(t, u)=0.1, \gamma_{2}(t, u)=0.3$; then the conditions in Theorem 7 are satisfied. According to Theorem 7, we know that $x(t)$ is permanent and $y(t)$ is extinct.

In Figure 1(b), we choose $\gamma_{1}(t, u)=0.3, \gamma_{2}(t, u)=0.3$; by Theorem 6 , we can obtain that both of $x(t)$ and $y(t)$ are extinct.

Then, we choose $\gamma_{1}(t, u)=0.1, \gamma_{2}(t, u)=0.1$, the values of $\sigma_{1}(t)$ and $\sigma_{2}(t)$ are different between Figures $1(\mathrm{c})$ and $1(\mathrm{~d})$.

In Figure $1(\mathrm{c})$, we choose $\sigma_{1}(t)=0.3, \sigma_{2}(t)=0.9$; then the conditions in Theorem 7 are satisfied. According to Theorem 7, we know that $x(t)$ is permanent and $y(t)$ is extinct.

In Figure $1(\mathrm{~d})$, we choose $\sigma_{1}(t)=0.8, \sigma_{2}(t)=0.9$; by Theorem 6, we get that both of $x(t)$ and $y(t)$ are extinct.

In Figure 2, we choose $\gamma_{1}=2.8, \gamma_{2}=2.3, \sigma_{1}(t)=$ $0.3, \sigma_{2}(t)=0.4, \gamma_{1}(t, u)=0.1$, and $\gamma_{2}(t, u)=0.1$; other parameters are the same as in Figure 1; according to Theorems 8 and 10 , we obtain that system (4) is permanent in mean and stochastically permanent.

In conclusion, permanence and extinction of the predator and the prey for the intensity of white noise and Lévy noise are given in Table 1.

In this paper, we consider a nonautonomous impulsive stochastic predator-prey system with Lévy jumps, which considers the predator is omnivorous. We prove that the system has a unique global positive solution. From Theorem 6 and Figure 1, we can know that if the intensities of white noise and Lévy noise are sufficiently large, then the species will be extinct (see Figures 1(b) and 1(d)). According to Theorems 8 and 10, we know that system (4) is permanent in mean and stochastically permanent under some conditions in Figure 2. The change of permanent conditions shows an 
TABLE 1: Permanence and extinction for different noise parameters.

\begin{tabular}{|c|c|c|c|c|}
\hline White noise $\sigma_{1}$ & White noise $\sigma_{2}$ & Lévy noise $\gamma_{1}$ & Lévy noise $\gamma_{2}$ & Permanence and extinction \\
\hline 0.3 & 0.4 & 0.1 & 0.3 & Permanence for $x$ and extinction for $y$ \\
\hline 0.3 & 0.4 & 0.3 & 0.3 & Extinction for $x$ and $y$ \\
\hline 0.3 & 0.9 & 0.1 & 0.1 & Permanence for $x$ and extinction for $y$ \\
\hline 0.8 & 0.9 & 0.1 & 0.1 & Extinction for $x$ and $y$ \\
\hline 0.3 & 0.4 & 0.1 & 0.1 & Permanence for $x$ and $y$ \\
\hline
\end{tabular}
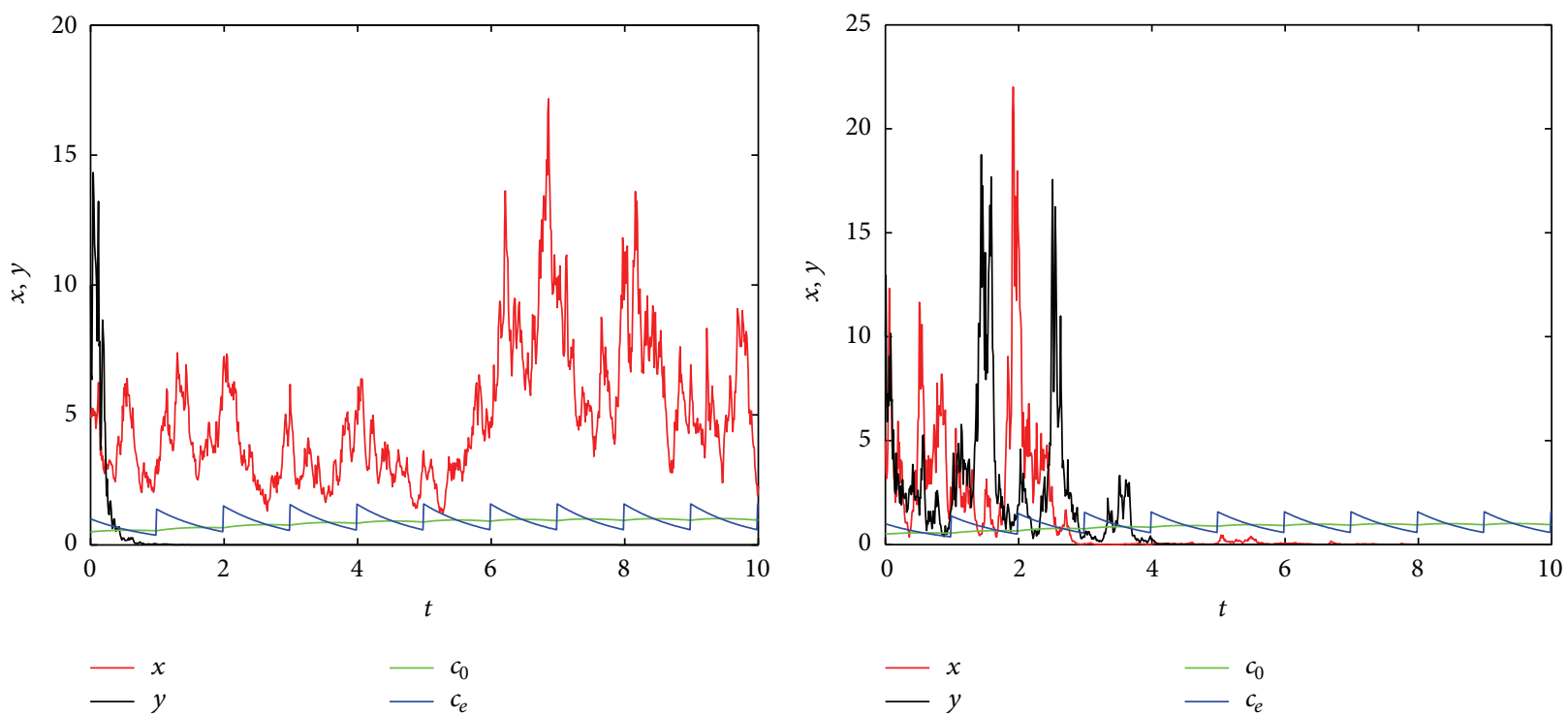

(a) $\sigma_{1}(t)=0.3, \sigma_{2}(t)=0.4 ; \gamma_{1}(t, u)=0.1, \gamma_{2}(t, u)=0.3$

(b) $\sigma_{1}(t)=0.3, \sigma_{2}(t)=0.4 ; \gamma_{1}(t, u)=0.3, \gamma_{2}(t, u)=0.3$
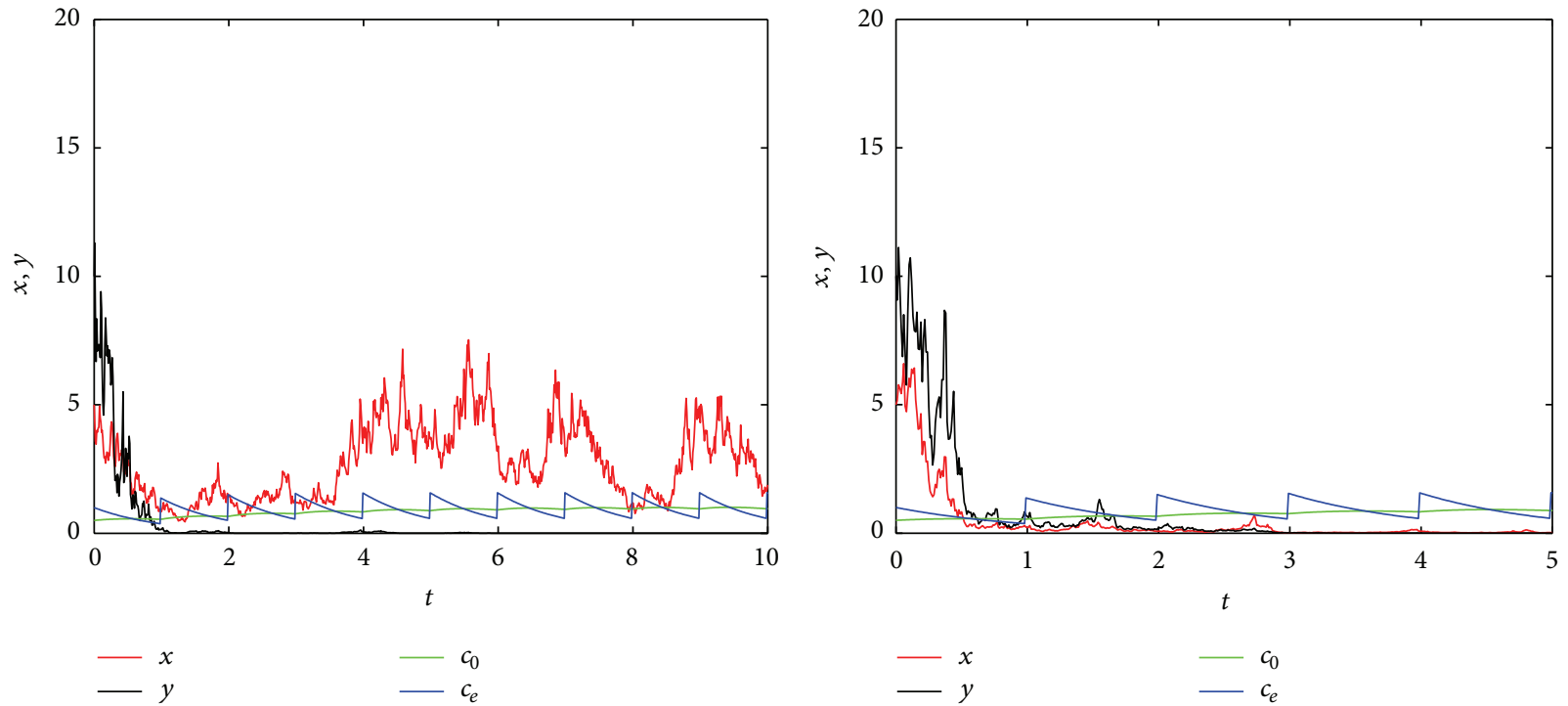

(c) $\sigma_{1}(t)=0.3, \sigma_{2}(t)=0.9 ; \gamma_{1}(t, u)=0.1, \gamma_{2}(t, u)=0.1$

(d) $\sigma_{1}(t)=0.8, \sigma_{2}(t)=0.9 ; \gamma_{1}(t, u)=0.1, \gamma_{2}(t, u)=0.1$

FiguRE 1: The red line and the black line represent $x(t)$ and $y(t)$, respectively. The initial value is $x(0)=5, y(0)=10, c_{0}(0)=0.5$, and $c_{e}(0)=1$. In (a), $x(t)$ is permanent and $y(t)$ is extinct; in (b), both of $x(t)$ and $y(t)$ are extinct. In (c), $x(t)$ is permanent and $y(t)$ is extinct; in (d), both of $x(t)$ and $y(t)$ are extinct. 


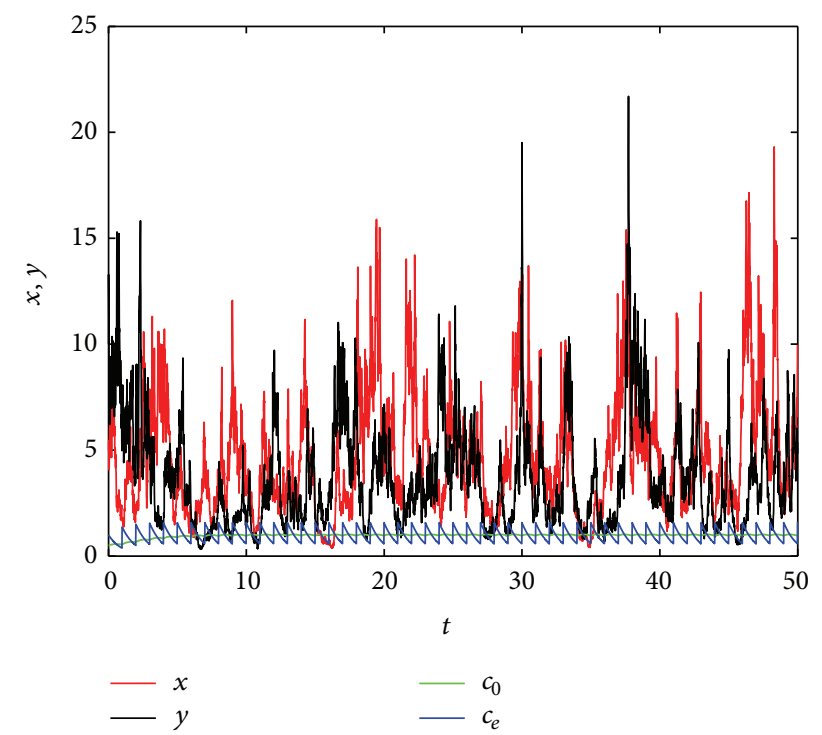

Figure 2: The red line and the black line represent $x(t)$ and $y(t)$, respectively. The initial value is $x(0)=5, y(0)=10, c_{0}(0)=0.5$, and $c_{e}(0)=1$. Both $x(t)$ and $y(t)$ are stochastically permanent when $\sigma_{1}(t)=0.3, \sigma_{2}(t)=0.4, \gamma_{1}(t, u)=0.1$, and $\gamma_{2}(t, u)=0.1$.

important property; that is, the permanence of species has a close relationship with the intensity of white noise and Lévy noise. Obviously, white noises and Lévy noises are harmful to the permanence of populations.

There are some investigations for stochastic epidemic models $[14,15]$. It is very significant for the natural world. Some interesting questions deserve further investigation. One could study more realistic but more complex models. Also it is interesting to investigate evolutionary dynamics of stochastic evolutionary model, and we leave these for future work.

\section{Appendix}

Proof of Lemma 5. (I) If $\langle\delta\rangle^{*} \geq 0, \int_{0}^{t} \delta(s) d s>0$ for $t$ large enough. By Assumption 3 and the strong law of large numbers for local martingales, one has

$$
\begin{aligned}
\lim _{t \rightarrow+\infty} \frac{1}{t} \sum_{i=1}^{2} \delta_{i} \int_{0}^{t} \int_{\mathbb{Y}} \ln \left(1+\gamma_{i}(u)\right) \widetilde{N}(d s, d u) & =0, \\
\lim _{t \rightarrow+\infty} \frac{B(t)}{t} & =0
\end{aligned}
$$

a.s.

Then, for arbitrary $\varepsilon>0$, there exists a $T_{1}>0$ such that for $t>T_{1}$

$$
\begin{aligned}
& \frac{1}{t}\left[\alpha B(t)+\sum_{i=1}^{2} \delta_{i} \int_{0}^{t} \int_{\mathbb{V}} \ln \left(1+\gamma_{i}(u)\right) \widetilde{N}(d s, d u)\right] \\
& \quad<\varepsilon .
\end{aligned}
$$

Define

$$
\varphi(t)=\int_{0}^{t} \eta(s) d s
$$

for $t \geq T_{1}$; then

$$
\begin{aligned}
\frac{d \varphi(t)}{d t} & =\eta(t) \\
\ln \frac{d \varphi(t)}{d t} & \leq \int_{0}^{t}(\delta(s)+\varepsilon) d s-\delta_{0} \varphi(t),
\end{aligned}
$$

for $t \geq \max \left\{T, T_{1}\right\}=T_{2}$.

Integrating the above inequality from $T_{2}$ to $t$ yields

$$
\begin{aligned}
\varphi(t) \leq & \frac{1}{\delta_{0}} \ln \left(e^{\delta_{0} \varphi\left(T_{2}\right)}+\delta_{0} \int_{T_{2}}^{t} e^{\int_{0}^{\theta}(\delta(s)+\varepsilon) d s} d \theta\right) \\
\leq & \frac{1}{\delta_{0}} \ln \left(e^{\delta_{0} \varphi\left(T_{2}\right)}+\delta_{0} e^{\int_{0}^{t}(\delta(s)+\varepsilon) d s}\left(t-T_{2}\right)\right) \\
\leq & \frac{1}{\delta_{0}} \int_{0}^{t}(\delta(s)+\varepsilon) d s \\
& +\frac{1}{\delta_{0}} \ln \left(\delta_{0}\left(t-T_{2}\right)+e^{\delta_{0} \varphi\left(T_{2}\right)}\right) .
\end{aligned}
$$

Dividing by $t$ and taking the superior limit of both sides of (A.5) lead to

$$
\langle\eta\rangle^{*} \leq \frac{\langle\delta\rangle^{*}+\varepsilon}{\delta_{0}} \quad \text { a.s. }
$$

By the arbitrariness of $\varepsilon$, one has $\langle\eta\rangle^{*} \leq\langle\delta\rangle^{*} / \delta_{0}$, if $\langle\delta\rangle^{*} \geq 0$. Moreover, if $\langle\delta\rangle^{*}<0$, we get

$$
\limsup _{t \rightarrow+\infty} \frac{1}{t} \ln \eta(t) \leq\langle\delta\rangle^{*}+\varepsilon<0,
$$

for $\varepsilon$ sufficiently small. Thus

$$
\lim _{t \rightarrow \infty} \eta(t)=0 \quad \text { a.s., if }\langle\delta\rangle^{*}<0 .
$$

(II) The proof of (II) is similar to (I). Here we omits the proof.

\section{Competing Interests}

The authors declare that they have no competing interests.

\section{Acknowledgments}

The study was supported by the National Natural Science Foundation of China (11371230), the SDUST Research Fund (2014TDJH102), Shandong Provincial Natural Science Foundation, China (ZR2012AM012, ZR2015AQ001), Joint Innovative Center for Safe and Effective Mining Technology and Equipment of Coal Resources, Shandong Province, a Project of Shandong Province Higher Educational Science, and Technology Program of China (J13LI05). 


\section{References}

[1] D. Mukherjee, "Persistence and global stability of a population in a polluted environment with delay," Journal of Biological Systems, vol. 10, no. 3, pp. 225-232, 2002.

[2] S. B. Hsu and T. W. Huang, "Global stability for a class of predator-prey systems," SIAM Journal on Applied Mathematics, vol. 55, no. 3, pp. 763-783, 1995.

[3] B. Liu and L. Zhang, "Dynamics of a two-species LotkaVolterra competition system in a polluted environment with pulse toxicant input," Applied Mathematics and Computation, vol. 214, no. 1, pp. 155-162, 2009.

[4] J. J. Jiao, W. Long, and L. S. Chen, "A single stage-structured population model with mature individuals in a polluted environment and pulse input of environmental toxin," Nonlinear Analysis: Real World Applications, vol. 10, no. 5, pp. 3073-3081, 2009.

[5] X. Z. Meng, Z. Li, and J. J. Nieto, "Dynamic analysis of Michaelis-Menten chemostat-type competition models with time delay and pulse in a polluted environment," Journal of Mathematical Chemistry, vol. 47, no. 1, pp. 123-144, 2010.

[6] R. Rudnicki and K. Pichór, "Influence of stochastic perturbation on prey-predator systems," Mathematical Biosciences, vol. 206, no. 1, pp. 108-119, 2007.

[7] X. R. Mao, S. Sabanis, and E. Renshaw, "Asymptotic behaviour of the stochastic Lotka-Volterra model," Journal of Mathematical Analysis and Applications, vol. 287, no. 1, pp. 141-156, 2003.

[8] M. Liu and K. Wang, "Extinction and permanence in a stochastic non-autonomous population system," Applied Mathematics Letters, vol. 23, no. 12, pp. 1464-1467, 2010.

[9] D. Q. Jiang, C. Y. Ji, X. Y. Li, and D. O'Regan, "Analysis of autonomous Lotka-Volterra competition systems with random perturbation," Journal of Mathematical Analysis and Applications, vol. 390, no. 2, pp. 582-595, 2012.

[10] J. Bao and C. Yuan, "Stochastic population dynamics driven by Lévy noise," Journal of Mathematical Analysis and Applications, vol. 391, no. 2, pp. 363-375, 2012.

[11] Q. Liu and Q. M. Chen, "Analysis of a stochastic delay predatorprey system with jumps in a polluted environment," Applied Mathematics and Computation, vol. 242, pp. 90-100, 2014.

[12] X. H. Zhang, W. X. Li, M. Liu, and K. Wang, "Dynamics of a stochastic Holling II one-predator two-prey system with jumps," Physica A: Statistical Mechanics and its Applications, vol. 421, pp. 571-582, 2015.

[13] M. Liu and K. Wang, "Stochastic Lotka-Volterra systems with Lévy noise," Journal of Mathematical Analysis and Applications, vol. 410, no. 2, pp. 750-763, 2014.

[14] Y. Zhao and D. Q. Jiang, "The threshold of stochastic SIRS epidemic model with saturated incidence," Applied Mathematics Letters, vol. 34, pp. 90-93, 2014.

[15] X. Z. Meng, S. N. Zhao, T. Feng, and T. H. Zhang, "Dynamics of a novel nonlinear stochastic SIS epidemic model with double epidemic hypothesis," Journal of Mathematical Analysis and Applications, vol. 433, no. 1, pp. 227-242, 2016. 


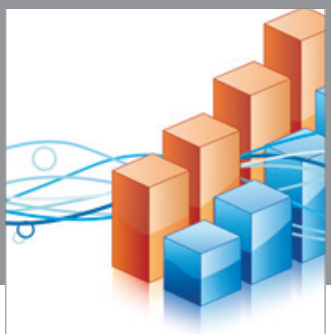

Advances in

Operations Research

vatem alat4

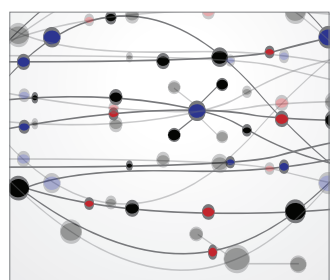

\section{The Scientific} World Journal
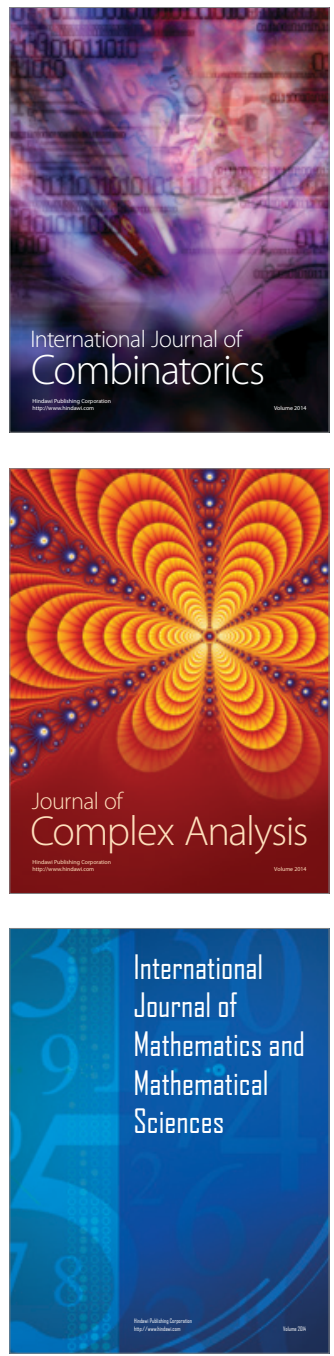
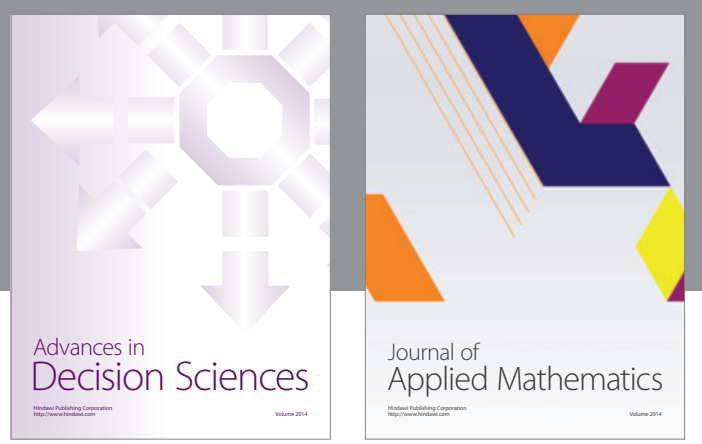

Algebra

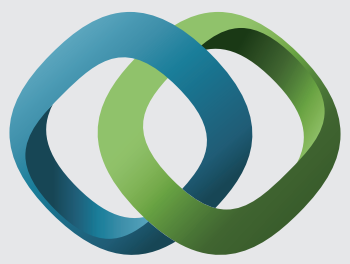

\section{Hindawi}

Submit your manuscripts at

http://www.hindawi.com
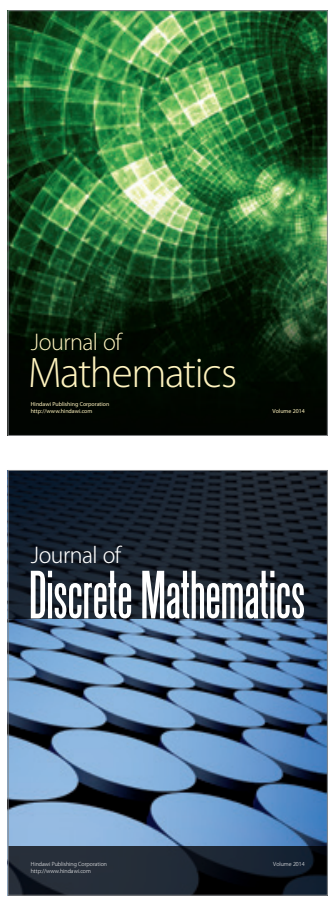

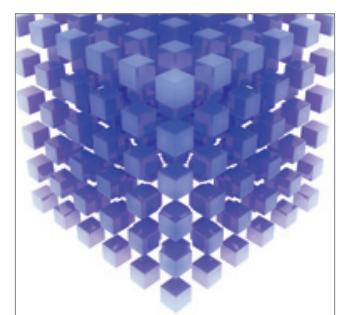

Mathematical Problems in Engineering
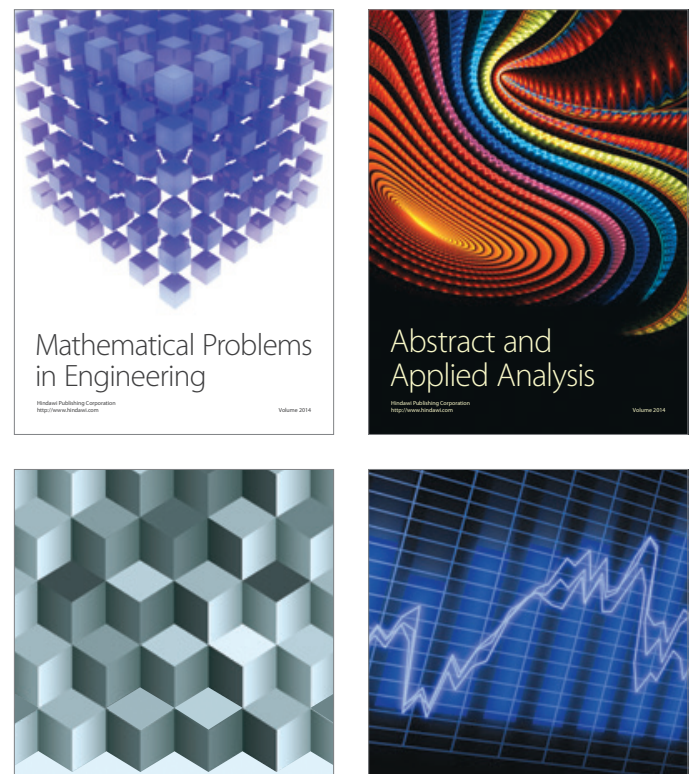

Journal of

Function Spaces

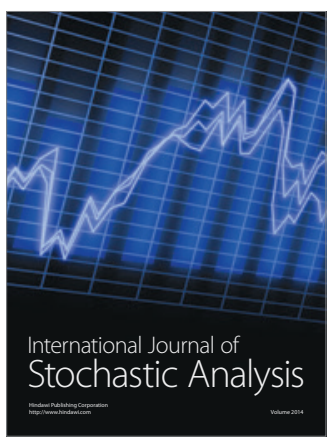

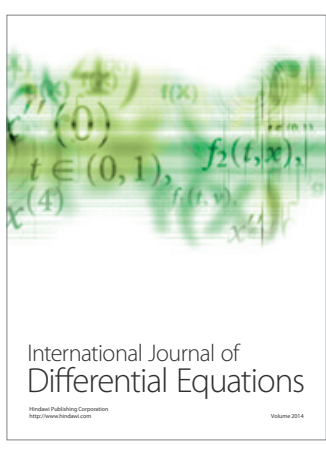
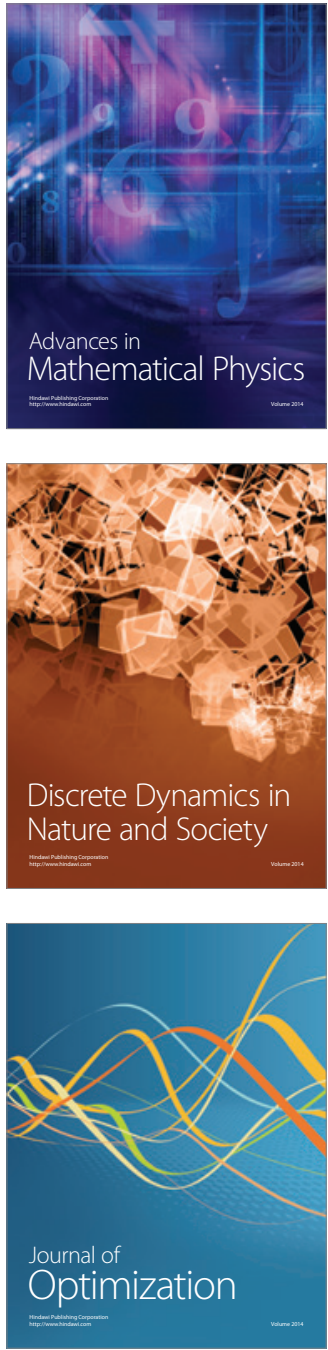